\title{
ANTIFERROMAGNETISM AND SUPERCONDUCTIVITY IN THREE-BAND HUBBARD MODEL
}

\author{
J. DutKa AND A.M. Oleś \\ Institute of Physics, Jagiellonian University, Reymonta 4, 30-059 Kraków, Poland
}

We determined the phase diagram of the three-band model for $\mathrm{CuO}_{2}$ planes of high temperature superconductors with an attractive interoxygen interaction $U_{p p}$. The found magnetic moments of $0.47 \mu_{\mathrm{B}}$ in the undoped system, the disappearance of antiferromagnetic order at the doping of $\delta \approx 0.06$ hole per $\mathrm{CuO}_{2}$ unit, as well as the enhanced tendency to superconductivity with the decreasing value of charge-transfer gap, are in good agreement with the experimental findings.

PACS numbers: 75.50.Ee, 74.20.-z

The Cu-based high temperature superconducting oxides (HTSO) are known to be insulators of a charge-transfer (CT) type, with the CT gap originating from the energy difference between the $\mathrm{O}\left(2 p_{\sigma}\right)$ and the $\mathrm{Cu}\left(3 d_{x^{2}-y^{2}}\right)$ orbitals [1]. The electronic states of these materials are therefore frequently described by the so-called three-band model which includes the above orbitals and local Coulomb interactions between the holes [2]. It is well established that the undoped materials, $\mathrm{La}_{2} \mathrm{CuO}_{4}$ and $\mathrm{YBa}_{2} \mathrm{Cu}_{3} \mathrm{O}_{6}$, exhibit the antiferromagnetic (AF) long range order (LRO) in their ground states, with the magnetic structures consisting of two sublattices [3, 4]. Unfortunately, the band structure calculations performed with the local density approximation (LDA) fail to reproduce the AF LRO in the undoped HTSO [1]. The doping of the parent compounds to $\mathrm{La}_{2-x} \mathrm{Sr}_{x} \mathrm{CuO}_{4}$ and $\mathrm{YBa}_{2} \mathrm{Cu}_{3} \mathrm{O}_{6+x}$, respectively, suppresses the AF LRO already when the concentration of holes within the $\mathrm{CuO}_{2}$ planes increases by a few percent and gives superconducting (SC) ground states for higher doping [4]. The transition temperature $T_{\mathrm{c}}$ to the latter state is strongly dependent on the applied pressure [5]. It is therefore of interest to investigate to what extent the three-band model may be used to account for these observed physical properties of the HTSO, and in particular for the coexistence of AF and SC states in the experimental phase diagrams $[3,4]$.

The Hamiltonian which describes holes in the $\mathrm{Cu}\left(3 d_{x^{2}-y^{2}}\right), \mathrm{O}\left(2 p_{x}\right)$ and $\mathrm{O}\left(2 p_{y}\right)$ orbitals, respectively, has the form [2], 


$$
\begin{aligned}
& H_{0}=\varepsilon_{d}^{0} \sum_{m \sigma} n_{d m \sigma}+\varepsilon_{p}^{0} \sum_{i \sigma} n_{p i \sigma}+V_{0} \sum_{\langle i m\rangle \sigma}(-1)^{\pi_{m i}}\left(d_{m \sigma}^{\dagger} a_{i \sigma}+\mathrm{H} . \mathrm{c} .\right) \\
& +t_{p p}^{0} \sum_{\langle i j\rangle \sigma}(-1)^{\gamma_{i j}}\left(a_{i \sigma}^{\dagger} a_{j \sigma}+\text { H.c. }\right)+U_{d} \sum_{m} n_{d m \uparrow} n_{d m \downarrow}+U_{p} \sum_{i} n_{p i \uparrow} n_{p i \downarrow} \\
& \quad+U_{d p} \sum_{\langle i m\rangle \sigma \sigma^{\prime}} n_{d m \sigma} n_{p i \sigma^{\prime}}+U_{p p} \sum_{\langle i j\rangle \dot{\sigma} \sigma^{\prime}} n_{p i \sigma} n_{p j \sigma^{\prime}}
\end{aligned}
$$

In Eq. (1) the vacuum is defined as filled $\mathrm{Cu} 3 d^{10}$ and $\mathrm{O} 2 p^{6}$ states, and the undoped state contains one hole per unit cell, $n=1$. Operators $d_{m \sigma}^{\dagger}$ and $a_{i \sigma}^{\dagger}$ create a hole of a spin $\sigma$ in an atomic $\mathrm{Cu}\left(3 d_{x^{2}-y^{2}}\right)$, and $\mathrm{O}\left(2 p_{x}\right)$ or $\mathrm{O}\left(2 p_{y}\right)$ orbital, respectively, with the energies $\varepsilon_{d}^{0}$ and $\varepsilon_{p}^{0}$ which define the CT energy, $\Delta=\varepsilon_{p}^{0}-\varepsilon_{d}^{0}$. The summations over $\langle m i\rangle$ and $\langle i j\rangle$ are restricted to nearest-neighbour $\mathrm{Cu}-\mathrm{O}$ and $\mathrm{O}-\mathrm{O}$ pairs and involve each pair only once, $V_{0}$ and $t_{p p}^{0}$ stand for the respective hybridization energies, and $\pi_{m i}$ and $\gamma_{i j}$ are the respective phase factors. The on-site and intersite Coulomb interactions are described by the elements $U_{d}, U_{p}$ and $U_{d p}$, respectively. The attractive oxygen-oxygen interaction $U_{p p}<0$ simulates the effects of other (out-of-plane) degrees of freedom which may be responsible for the pairing in HTSO.

In order to get a qualitative insight into the physical properties of the holes within $\mathrm{CuO}_{2}$ planes and to calculate the correlated ground state, we use first the HFA,

$$
\begin{aligned}
H_{\mathrm{HFA}} & =\sum_{m \sigma}\left(\varepsilon_{d}-\sigma v \mathrm{e}^{\mathrm{i} Q \boldsymbol{Q R}}\right) n_{d m \sigma}+\varepsilon_{p} \sum_{i \sigma} n_{p i \sigma} \\
& +\sum_{\langle i m\rangle \sigma} V_{\sigma}(-1)^{\pi_{m i}}\left(d_{m \sigma}^{\dagger} a_{i \sigma}+\text { H.c. }\right) \\
& +t_{p p} \sum_{\langle i j\rangle \sigma}(-1)^{\gamma_{i j}}\left(a_{i \sigma}^{\dagger} a_{j \sigma}+\text { II.c. }\right) \\
& +U_{p p} \sum_{\langle i j\rangle \sigma}\left(a_{i \sigma}^{\dagger} a_{j-\sigma}^{\dagger}\left\langle a_{j-\sigma} a_{i \sigma}\right\rangle_{0}+\text { H.c. }\right),
\end{aligned}
$$

where $\varepsilon_{d}$ and $\varepsilon_{p}$ are the effective HF levels,

$$
\begin{aligned}
& \varepsilon_{d}=\varepsilon_{d}^{0}+\frac{1}{2} U_{d} n_{d}+4 U_{d p} n_{p}, \\
& \varepsilon_{p}=\varepsilon_{p}^{0}+\left(\frac{1}{2} U_{p}+4 U_{p p}\right) n_{p}+2 U_{d p} n_{d},
\end{aligned}
$$

which define the HF value of the CT energy, $\Delta_{\mathrm{HF}}=\varepsilon_{p}-\varepsilon_{d}$. The hopping elements $V_{\sigma}$ and $t_{p p}$ are similarly renormalized by the respective Fock terms. Here $n_{d}=$ $\sum_{\sigma}\left\langle n_{d m \sigma}\right\rangle_{0}$ and $n_{p}=\sum_{\sigma}\left\langle n_{p i \sigma}\right\rangle_{0}$ are the average $\mathrm{Cu}$ and $\mathrm{O}$ occupancies by holes. All averages above, $\langle\ldots\rangle_{0}$, are determined in the Hartree-Fock (HF) ground state. $v$ is the AF potential which in the $\mathrm{HF}$ approximation fulfils the equation $v=\frac{1}{2} U m$, with $m$ being the magnetic moment, and takes lower values in the correlated states introduced below. $Q=(\pi / a, \pi / a)$ is the wave vector corresponding to extra periodicity due to the AF ordering. The ground state of the system can be found by minimizing the energy $E_{0}=\left\langle H_{\mathrm{HFA}}\right\rangle_{0}$ with respect to the AF and SC order parameters, $m$ and $\xi$, respectively. The first one is the amplitude of the spin 
density wave at $\mathrm{Cu}$ sites, $\left\langle n_{d m \sigma}\right\rangle_{0}=\left[n_{d}+\sigma m \exp \left(\mathrm{i} Q \boldsymbol{R}_{\mathrm{m}}\right)\right] / 2$, while the second one is proportional to the SC correlations $\left\langle a_{j, \sigma}^{\dagger} a_{i,-\sigma}^{\dagger}\right\rangle_{0}$.

Next we have obtained the correlated $\mathrm{AF}$ ground states by making use of the local ansatz (LA) method in which the ground state reads as follows [6]:

$$
\left|\psi_{0}\right\rangle=\exp \left(-\sum_{n} \eta_{n} O_{n}\right)\left|\phi_{0}\right\rangle
$$

where $\left|\phi_{0}\right\rangle$ is the respective HF state and $\eta_{n}$ are the variational parameters. $O_{n}$ are the local operators which optimize the probability of two holes occupying the same orbital $\left(O_{m}^{(1)}=n_{m \uparrow} n_{m \downarrow}\right.$ and $\left.O_{i}^{(1)}=n_{i \uparrow} n_{i \downarrow}\right)$ and two orbitals at the nearest-neighbour sites $\left(O_{m i}^{(2)}=\sum_{\sigma \sigma^{\prime}} n_{m \sigma} n_{i \sigma^{\prime}}\right)$, respectively, as well as renormalize the density distribution by the density operators $\left(O_{m}^{(0)}=\sum_{\sigma} n_{m \sigma}\right)$. The correlation energy and the variational parameters are determined by performing a local cluster expansion. More details may be found in Ref. [6].

The parameters of the three-band model (1) for $\mathrm{CuO}_{2}$ planes were determined from LDA calculations [7, 8]; here we have used a representative set of Ref. [7]: $\Delta=\varepsilon_{p}^{0}-\varepsilon_{d}^{0}=3.5 \mathrm{eV}, V_{0}=1.3 \mathrm{eV}, t_{p p}^{0}=0.65 \mathrm{eV}, U_{d}=10 \mathrm{eV}, U_{p}=4 \mathrm{eV}$, $U_{d p}=1.2 \mathrm{eV}$, and $U_{p p}=0$, which gives an $\mathrm{AF}$ ground state of the undoped $\mathrm{CuO}_{2}$ plane, with the magnetic moment of $0.7 \mu_{\mathrm{B}}$. This mean-field value is renormalized downwards to $0.47 \mu_{\mathrm{B}}$, if the same reduction of the moment due to quantum fluctuations as that for a two-dimensional Heisenberg antiferromagnet (by $39.6 \%$ ) is included. This latter value agrees very well with the moment of $0.55 \mu_{\mathrm{B}}$ measured in $\mathrm{La}_{2} \mathrm{CuO}_{4}$ [9]. Similarly, if a smaller reduction of $27 \%$ of the mean-field value corresponding to a system of two planes is assumed, one finds a moment of $0.56 \mu_{\mathrm{B}}$ which agrees well with the measured $0.65 \mu_{\mathrm{B}}$ for a two-plane AF state in $\mathrm{YBa}_{2} \mathrm{Cu}_{3} \mathrm{O}_{6}$ [4].

The magnetic moment $m$ and the local moment at $\mathrm{Cu}$ sites,

$$
\left\langle\left(S_{m}^{z}\right)^{2}\right\rangle=\frac{\left\langle\psi_{0}\left|\left(S_{m}^{z}\right)^{2}\right| \phi_{0}\right\rangle}{\left\langle\psi_{0} \mid \phi_{0}\right\rangle},
$$

obtained for the correlated ground states of the doped system are presented in Fig. 1. The AF LRO disappears at rather small doping of ; $=0.06(\delta=n-1)$ hole per unit cell, in qualitative agreement with the experimental phase diagrams $[3,4]$. The local moments survive in the correlated nonmagnetic states and demonstrate strong localization of holes at $\mathrm{Cu}$ sites. They agree with the results of variational Monte-Carlo calculations and with the experimental observations of short-range AF order, as discussed in more detail in Ref. [6].

For higher doping $\delta$ and for sufficiently large attractive interaction $U_{p p}$ one finds SC ground states with $\xi \neq 0$, separated from the AF states. The analysis of these states was made: (i) to establish the necessary combination of doping, $\delta$, and of the interoxygen attraction, $U_{p p}$, to produce SC pairs in the present strongly correlated system, and (ii) to study the dependence of the CT energy gap, $E_{\mathrm{g}}$, on pressure. For a qualitative discussion we use the $\mathrm{HF}$ approximation which gives the phase diagram presented in Fig. 2. The AF order which disappears with doping of $\delta=0.44$ holes per $\mathrm{CuO}_{2}$ unit at $U_{p p}=0$. Thus, the tendency to antiferromagnetism is overestimated due to the neglect of local hole correlations 


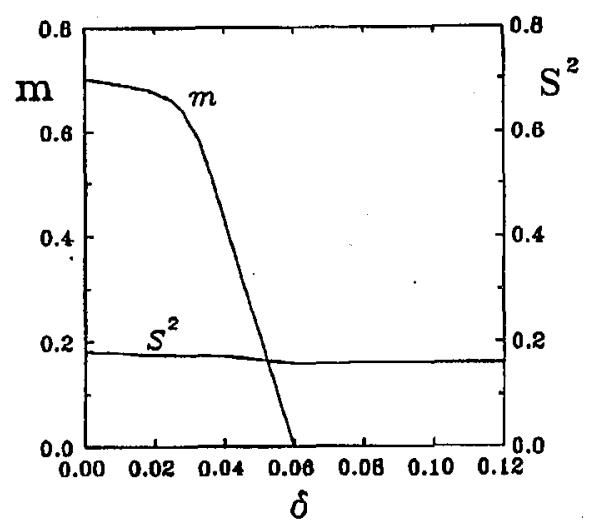

Fig. 1. The AF order parameter $m$ and the local moment $\left\langle\left(S_{m}^{z}\right)^{2}\right\rangle$ as functions of doping by holes $\delta$. The parameters are taken from Ref. [7]: $\Delta=3.5 \mathrm{eV}, V_{0}=1.3 \mathrm{eV}$, $t_{p p}^{0}=0.65 \mathrm{eV}, U_{d}=10 \mathrm{eV}, U_{p}=4 \mathrm{eV}, U_{d p}=1.2 \mathrm{eV}$.

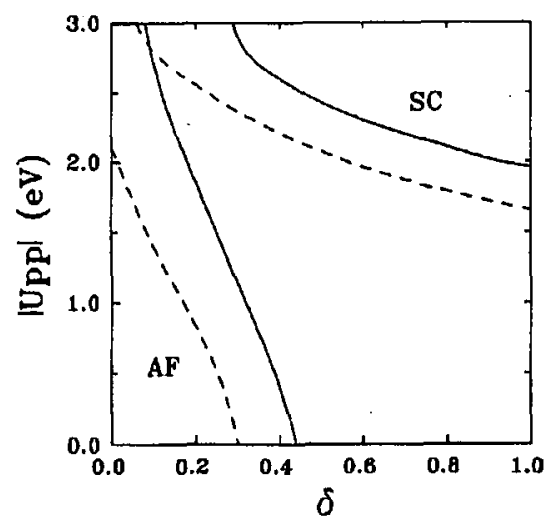

Fig. 2. Regions of stability of antiferromagnetic (AF) and superconducting (SC) phase in the $\left(U_{p p}, \delta\right)$ plane for $\Delta=3.5 \mathrm{eV}$ (full lines) and $\Delta=2.0 \mathrm{eV}$ (dashed lines). The other parameters as in Fig. 1.

which lead to the formation of local moments. It is reasonable to assume that the effective interoxygen attraction increases with doping by holes. As seen in Fig. 2, the AF phase is then gradually suppressed which shows the competition between the two considered ordered states.

Finally, we examine the effect of pressure on the system. It is known from experiment [5] that the cuprates exhibit quite different behaviour, depending on the oxygen coordination. On one hand, for nonsuperconducting $T^{\prime}$ and $T^{*}$ phase cuprates, i.e. with four and five oxygens around each in-plane copper ion, respectively, $E_{\mathrm{g}}$ increases with applied pressure. On the other hand, for $T$-phase cuprates (with six oxygens surrounding each in-plane copper ion, as in $\mathrm{La}_{2} \mathrm{CuO}_{4}$ ) $E_{\mathrm{g}}$ decreases with pressure which gives a negative pressure coefficient of the CT gap. In 
the model we simulate the effect of pressure in $\mathrm{CuO}_{2}$ planes by decreasing $\Delta_{\mathrm{HF}}$. In Fig. 2 we present $\left(U_{p p}, \delta\right)$ phase diagrams with substantially different values of $\Delta_{0}=3.5 \mathrm{eV}$ and $2.0 \mathrm{eV}$, corresponding to $\Delta_{\mathrm{HF}}=1.2 \mathrm{eV}$ and $-0.1 \mathrm{eV}$, respectively. The region of stability of AF (SC) phase diminishes (grows) with decreasing $\Delta$. This effects are associated with the hole redistribution towards oxygens, while $\Delta$ is decreased. The effect is in qualitative agreement with the experimental findings, though values of $U_{p p}$ required to produce paired state seem to be unrealistically large.

It is remarkable that the effective attractive interactions required for the stabilization of local SC correlations are of the order of half of the respective (oxygen) bandwidth, similarly as in the extended Hubbard model [10]. In fact, one expects that the hopping parameter, $t_{p p}$, as well as the hybridization element, $V_{0}$, are strongly renormàlized by electron-phonon interaction and, therefore, already much smaller effective interoxygen attractive interactions might suffice to stabilize the SC ground state. Although the obtained phase diagrams agree qualitatively with the ones observed in HTSO, the found large values of doping required to stabilize the $\mathrm{SC}$ states $(\delta \approx 0.3)$ suggest that purely interoxygen attractive interactions cannot explain the superconductivity in these materials. Thus we conclude that the SC ground states of HTSO are likely to be stabilized by a superposition of attractive interactions at both $\mathrm{Cu}$ and $\mathrm{O}$ sites. It remains to be investigated whether the copper-copper attractive interactions are induced by magnetic interactions, charge fluctuations, or by phonons. We believe that the presented qualitative model of pairing due to the interoxygen attraction may be considered as a generic model which, after its appropriate extension and more sophisticated treatment, may be successfully applied for the description of superconductivity in HTSO.

We acknowledge the financial support by the Committee for Scientific Research project No. 203869101.

\section{References}

[1] K.C. Haas, in: Solid State Physics, Vol. 42, Eds. H. Ehrenreich, D. Turnbull, Academic Press, Orlando 1989, p. 213.

[2] C.M. Varma, S. Schmitt-Rink, E. Abrahams, Solid State Commun. 62, 681 (1987); V.J. Emery, Phys. Rev. Lett. 58, 2794 (1987).

[3] Y. Kitaoka, K. Ishida, T. Kobayashi, K. Amaya, K. Asayama, Physica C 153-155, 733 (1988).

[4] J.M. Tranquada, A.H. Moudden, A.I. Goldman, P. Zolliker, D.E. Cox, G. Shirane, S.K. Sinha, D. Vaknin, D.C. Johnston, M.S. Alvarez, A.J. Jacobson, Phys. Rev. B 38, 2477 (1988).

[5] Y. Tokura, K. Kikuchi, T. Arima, T. Uchida, Phys. Rev. B 45, 7580 (1992).

[6] J. Dutka, A.M. Oles, Phys. Rev. B 43, 5622 (1991).

[7] M.S. Hybertsen, M. Schlüter, N.E. Christensen, Phys. Rev. B 39, 9028 (1989).

[8] J.B. Grant, A.K. McMahan, Phys. Rev. B 46, 8440 (1992).

[9] R.J. Birgeneau, D.R. Gabbe, H.P. Jenssen, M.A. Kastner, P.J. Picone, T.R. Thurston, G. Shirane, Y. Endoh, M. Sato, K. Yamada, Y. Hidaka, M. Oda, Y. Enomoto, M. Suzuki, T. Murakami, Phys. Rev. B 38, 6614 (1988).

[10] R. Micnas, J. Ranninger, S. Robaszkiewicz, Rev. Mod. Phys. 62, 113 (1990). 